\title{
Simplicity Rules the Roost: Exploring Birdbrain Parental Investment Heuristics
}

\author{
Seth Bullock, Jennifer Nerissa Davis and Peter M. Todd \\ Center for Adaptive Behavior and Cognition \\ Max Planck Institute for Human Development \\ Lentzeallee 94, D-14195 Berlin, Germany
}

\begin{abstract}
Parents raising multiple offspring must decide how to divide resources between them. Much empirical data on the parenting behaviour of particular species has been collected. Birds, in particular, have been shown to follow a number of provisioning rules. However, the adaptive significance of this variation in decision-making strategies has been largely unexplored. Here we present a simulation model of the western bluebird, Sialia mexicana, with which we explore the utility of various simple feeding heuristics. The simulated parents face the task of simultaneously raising several offspring who are of differing ages and thus have differing resource needs. We show that the success of simple rules of thumb varies with environmental parameters in a manner which (i) predicts experimental results in the biology literature, and (ii) can be explained using a notion of parental egalitarianism.
\end{abstract}

A repeated finding within the field of artificial life has been that complex adaptive behaviour may arise from the interaction between simple systems and their environment. This perspective contrasts starkly with received wisdom within the decision-making literature where simplicity is often automatically equated with stupidity. The reason for this prejudice is easy to see. The normative theories which currently dominate research into decision making (probabilistic reasoning, Bayesian reasoning, various propositional logics, etc.) often involve the complex integration of many pieces of information and the construction of elaborate models.

This complexity is geared towards achieving decision-making's gold standard: rational coherence. The proponents of these complex normative models claim that if one follows their prescriptions, and the assumptions that these models require in order to work properly are met, one is sure never to behave intransitively, never to hold inconsistent beliefs, and never to fall foul of reasoning fallacies or other delusions. In contrast, through his simplicity, the naive roulette-wheel gambler who bets his last dime on red thirteen because it has not come up for the entire time he has been sitting at the table, or simply because it is his lucky number, is revealing himself to be irrational and thus exploitable.

The appeal of rationality is very strong, and the failure of human and animal decision-making to live up to its high standards has been the cause of some consternation amongst psychologists and economists [1]. However, two recent 
research movements have challenged the rationality-based hegemony and suggested that successful, adaptive behaviour in the real world may not require the complex machinery advocated by the orthodoxy, but may rely on simple heuristics which although limited in their ability, are suited to the environments in which they must operate.

The first of these research programmes has become associated with the behaviour-based robotics field as it has tried to extricate itself from good oldfashioned artificial intelligence (GOFAI) [2]. The second is exemplified by decisionmaking researchers who are beginning to question the benefit of rational coherence if it can only be obtained at the expense of tractability and psychological reality [3].

The animal behaviour literature has proved influential in both of these cases. Roboticists have discovered that organisms have achieved robust, simple solutions to many of the problems they face as engineers, and that these natural solutions reveal that the elaborate planning and modelling which GOFAI assumes goes on inside the heads of even the simplest intelligent agents is to a surprising extent unnecessary [4]. Similarly, decision-making theorists have begun to appreciate that ethologists have had considerable success explaining animal behaviour from a perspective which appreciates that evolution will favour accurate and reliable reasoning, but accepts that this reasoning must be carried out in the real world by real mechanisms [5].

Here we approach an ecologically relevant decision-making task which is typically regarded as intractable from the perspective of normative frameworks and demonstrate that simple ecologically sound rules of thumb can achieve high levels of performance and that their performance predicts empirical data collected by animal behaviour researchers.

\section{Parental Investment}

A decision-making problem which besets all animals which care for multiple simultaneous young is how to divide resources amongst these offspring. A classical approach to this problem would be to treat it as a game against nature in which the parent must play a strategy which maximises return on investment over the period of care. However, since the moves in such a game are many and the options available at each move are plural, the game tree which must be analysed in order that such an approach lead to a solution is prohibitively large.

For example, Becker [6] provides an economic analysis of how rational human parents should distribute investment among their children, assuming that parents are trying to maximise aggregate child quality as defined by the sum of all the children's wealth as adults. This quality is a function of the resources invested in the child, the child's own skill and abilities, and any extra income he or she might earn through sheer luck. Becker assumes that there are diminishing returns on parental investment, and shows that as long as these diminishing returns are the same for all children, parents should distribute investment such that each child achieves the same degree of wealth. However, if some children 
are capable of accumulating more wealth per unit of parental investment than others, then parents should of course favour them.

While these conclusions may sound reasonable in general, they are of limited use in making predictions about actual parental behaviour in specific situations. This is because they assume that parents have some means of calculating the effects of each unit of investment on the future payoff they expect to gain from a child. In practice, however, this calculation can require involved manipulations of information that is itself difficult to obtain. Children do not come equipped with investment meters for their parents' convenience.

In response to problems of this kind, biological models of parental investment have only dealt either with single offspring at a time, multiple simultaneous offspring assumed to be identical, or have been limited to cases where parents are assumed to base individual investment decisions on fully informative offspring solicitation signals [7-9]. In addition, previous models have treated parental investment as a series of events that have independent consequences for offspring fitness [10].

These simplified models of the parental investment problem have identified two simple rules which animals can employ in order to successfully raise as many offspring to reproductive age as possible. When each offspring requires the same amount of investment in order to reach a given level of fitness, then one parental solution would be to treat each on the basis of its need. In birds, for example, chicks often beg for food when hungry. If intensity of begging is an honest and accurate signal of need, parents would then be expected to feed their chicks according to this intensity in order to achieve investment equality. This is clearly a very simple decision rule.

Alternatively, in the case that offspring are not born simultaneously (chicks, for example, often hatch at different times), behavioural ecologists have identified a second simple but adaptive parental decision rule: satisfy the oldest offspring first. Since one major predictor of the probability of survival to adulthood is the current age of offspring (the older they are, the closer they are to independence and reproduction, and the more likely they are to make it all the way there), parents are expected to benefit from preferentially investing in their older offspring [7].

However, birds display a variety of parental feeding patterns. Coots preferentially feed the smallest chicks[11]. Pigeons preferentially feed the hungriest [12]. Common swifts preferentially feed their largest/oldest ${ }^{1}$ chicks [13]. Fieldfares appear to feed randomly [14]. Despite the amount of published data on this topic, there has been no proposal for why such a variety of strategies should exist.

\section{Simulating Parental Investment}

Here we model the problem of parental investment as a series of interdependent food-allocation decisions, in which investment is meted out in many small indi-

\footnotetext{
${ }^{1}$ Feeding the largest of a brood of asynchronously hatched chicks is equivalent to feeding the oldest since it ensures that the oldest chick will remain the largest.
} 
visible doses. Since offspring are of different ages they will have different resource demands. A successful parental investment strategy must balance these demands over the care-giving period in an effective manner despite the stochasticity of the foraging environment. The model we present is an iterative computer simulation that mimics the feeding, digestion, metabolism, and growth of asynchronously hatched western bluebird (Sialia mexicana) chicks from hatching until fledging. This model allows us to explore the effects of important aspects of parental decision making which are difficult to capture with more formal approaches. More specifically, it allows us to explore the complex relationship between a proximal decision-rule and the ultimate effects of this rule.

We compared the performance of the various parental strategies reported in the parental investment literature and some which were not (i.e., preferentially feeding the youngest or feeding in a fixed random order), in terms of number and weight of chicks fledged, across a range of different artificial environments. In doing so we assume that begging is an honest signal of need [9] and hence that preferentially feeding the chick begging with the greatest intensity is equivalent to preferentially feeding the hungriest chick. $^{2}$

We chose to model western bluebirds because there is information available about both metabolic rates and growth rates for this species. Equations for metabolic rate, digestion rate, and stomach size of our simulated chicks were derived from published field data on growth and metabolic rates of bluebird chicks across the nestling period [15]. Values for the calorie content of the insects that parents feed to their chicks, and the proportion of metabolisable energy these items contain were taken from Dykstra \& Karasov [16].

To mimic environmental differences in food availability, we varied the frequency with which food was found and returned to the nest, using a parameter $p$ governing the probability of repeatedly finding food and $q$ governing the probability of repeatedly failing to find food. The decision rules we tested determined which chick was fed on any given occasion, and it is the sum of these feeding decisions over the course of the nestling period that determines overall parental success in terms of the total size of all fledged chicks.

A total of four simulated chicks hatched at one day intervals, were fed for 20 days, then fledged. Each 24-hour day was divided into 10-minute intervals of simulated time, during which: 1 . Any egg due to hatch, hatched. 2. A bug (food) was found by the foraging parent with probability determined by environmental parameters $p$ and $q$. 3. If a bug was found, the parent's decision strategy was used to choose a chick to feed. 4 . If the chosen chick had enough space in its stomach for the bug, it was fed, otherwise the next most preferred chick was chosen. 5. If a chick was fed, the food was added to the chick's stomach. 6. Each chick with food in its stomach gained calories by digesting 10 minutes' worth of that food. 7. Every chick burned 10 minutes' worth of calories in accordance

\footnotetext{
${ }^{2}$ In reality, rather than always honestly revealing their true level of need, chick begging strategies will have coevolved with parental provisioning strategies. Although modelling this coevolutionary process is beyond the scope of the present paper, it will be pursued in further work.
} 


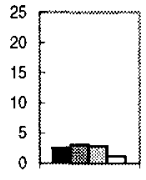

$10 \%-19 \%$

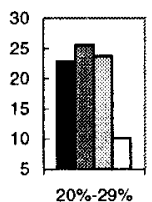

$20 \%-29 \%$

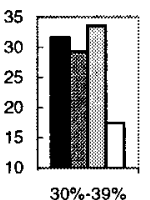

$30 \% \cdot 39 \%$

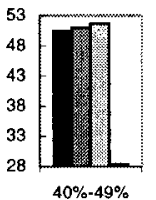

$40 \%-49 \%$

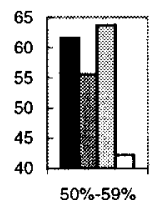

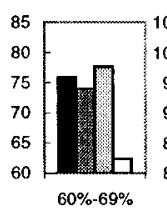

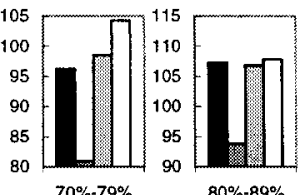

Fig. 1. The mean nest weight in grams achieved by each of four simple strategies (Random, Largest, Smallest, Hungriest, respectively) across eight classes of environment. Each strategy was simulated 500 times in each of 100 environments $\{p, q\}$ with parameters drawn from the set $\{.0, .1, \ldots, .9\}$. These environments are grouped by food availability, defined as the mean percentage of successful foraging attempts. Small differences in strategy successes can be very important ones. A half gram difference in the weight of a fledged chick is a significant one. The results depicted remain qualitatively the same for clutches of 3 or 5 chicks.

with its metabolic rate, gained or lost weight according to the net calorie change, and if it grew, its stomach capacity increased to accord with its new size. 8. If a chick's weight dropped below a certain age-specific limit it died. Foraging and feeding took place for 14 hours out of every day. As with real bluebirds, during the night no food was gathered or distributed, but, chicks continued to undergo steps 6 through 8 .

\section{Results}

Not surprisingly, the number of chicks that successfully fledged increased with increasing food availability. More interesting was the finding that the mean amount of food available to parents had a strong effect on the success of the different feeding rules (Fig. 1). Each of the four decision rules identified in the parental investment literature on birds was successful for some range of environmental "richness". Furthermore, the manner in which the success of these strategies varied with the abundance of food in the foraging environment is explicable from a perspective of "parental egalitarianism".

For relatively poor environments in which food was found $30 \%$ of the time or less, preferentially feeding the largest/oldest chick was the most successful decision rule. This rule, which picks out a single chick and targets it for preferential investment, does particularly well in environments where only a single chick can be raised. In this sense it is non-egalitarian, rejecting impartiality in favour of inequitable prejudice. In harsh environments, this bias is adaptive and successful.

For environments in which between $30 \%$ and $70 \%$ of parental foraging trips were successful, preferentially feeding the smallest chicks outperformed all other strategies. This strategy is more egalitarian than feeding the largest/oldest because the smallest chick is not fixed, but may change over time dependent on who gets fed. If whichever chick is currently smallest is preferentially fed, no smallest chick is likely to remain the smallest for long. In this way parents may 
achieve a limited form of equality amongst the chicks without any complicated calculation. Parents using this strategy are letting their environment (in this case the metabolism of their young) do some of the decision-making for them.

For richer environments, in which food is found on between $70 \%$ and $90 \%$ of foraging trips, the most successful parents fed on the basis of short term need, i.e., they fed the hungriest chicks, as revealed by intensity of begging. Preferentially feeding the hungriest chicks is an even more egalitarian strategy than favouring the smallest. Since the degree of hunger changes rapidly within a nest, if parents have access to some accurate indicator of this information, and the environment in which they forage is rich enough to provide for an entire brood, it makes sense to use this information in order to evenly balance the distribution of resources within the nest.

Finally, when food was even more abundant, all decision rules performed equally well. In such cases we would expect random feeding to evolve since this may make less demands on parental decision-making mechanisms.

Preferentially feeding the youngest chicks and feeding chicks in a fixed random order were never the most successful strategies. Thus our simulation agrees with empirical findings insofar as only the empirically observed strategies are the most successful in any simulated environment. But to what extent do our results concerning the manner in which the success of these strategies varies with environmental richness also agree with experimental and observational data?

The few published reports of species switching provisioning rules as a result of changing environmental conditions are surprisingly consistent with the findings of the simulation model. For instance, pied flycatcher females preferentially feed the smallest chicks under normal food conditions, but when food availability is experimentally reduced they preferentially feed the largest [17]. When food is plentiful, sparrow hawk mothers allocate food resources equally among chicks, when it is scarce they switch to a strategy that favours the largest [18].

\section{Combining Cues}

All of the simple strategies explored above make decisions based only on a single cue (weight, hunger, or age). As field studies suggest that parents sometimes combine cues [19], we also tested two sets of multi-cue feeding rules. Both sets fed chicks in an order determined by a criterion constructed as a linear weighted sum of the three chick cues (weight, hunger, age). The first set of rank-based multi-cue strategies calculated this criterion on the basis of a chick's rank-order within the nest. For every feeding decision made by a parent bird, all chicks were ranked according to each cue, and a chick's ranks were summed after being weighted according to the parental strategy. The parent then preferentially fed chicks scoring highest on this combined cue criterion.

The second set of real-valued multi-cue strategies weighted and summed the real cue values (e.g., weight in grams) rather than merely the chicks' ranks. We assessed both sets of strategies bearing in mind that the real values employed by 

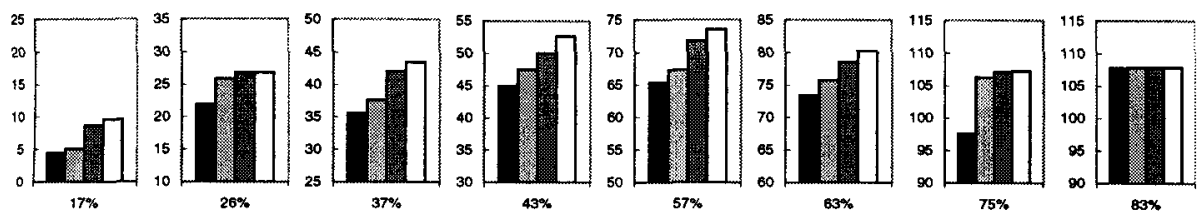

Fig. 2. The mean nest weight (in grams) achieved by the best of the 2197 possible mixed-cue strategies with cue weights drawn from the set $\{-6,-5, \ldots 5,6\}$. Each strategy was simulated 100 times in each of eight selected environments differing in mean food availability, but not variance. The performance of the best simple decision rule and of random feeding are shown for comparison. Columns show Random, Best Simple, Best Linear Weighted Ranks, and Best Linear Weighted Reals, respectively.

the second set of strategies might not be realistically assessable by parent birds, whereas ascertaining rank-orders is more likely to be within their ability.

Not surprisingly, for each of the environments tested, there was some subset of both the rank-based and real-valued mixed-cue rules that outperformed the single-cue rules (Fig. 2). Whilst the best real-valued strategies also outperformed the most successful of their rank-based cousins, they did not differ qualitatively in that they employed roughly the same weights. Furthermore, the degree of egalitarianism exhibited by the most successful mixed-cue rules increased with environmental quality just as it had done for the single-cue rules.

The strategies employed by the best multi-cue decision-makers can, for the most part, be regarded as refinements of those employed by the best one-cue decision-makers. In poor environments strategies weighted Age positively. In rich environments Hunger was weighted positively. Intermediate environments favoured strategies which either favoured small chicks, or achieved an intermediate degree of egalitarianism through balancing opposing cues (e.g., feeding young and heavy chicks). The ability of multi-cue strategies to balance opposing cues in this way can be used to explain the increased performance of these strategies.

\section{Optimal Investment?}

So far we have used the performance of random feeding behaviour as a lower bound against which to compare the utility of the parental investment strategies we have assessed. However, without knowing the maximum possible success that is attainable in each environment, it is difficult to assess how well these decision rules perform in an absolute sense. As was noted during the discussion of parental investment modelling, in practice finding this upper bound on performance is intractable. We have attempted to approximate the results of optimal investment by assessing feeding rules that integrate as much relevant information into their decisions as is computationally tractable, whether or not such strategies would be realistically achievable by parent birds. 
We implemented three short-term optimisation strategies and assessed their performance across the same range of environments used in the previous simulations. Each optimisation strategy relied on knowledge of the equations underlying chick metabolism and growth, the character of the stochasticity in the environment, and the exact state of each chick in the nest. These strategies then utilised this knowledge to make reasonable guesses about the future and thereby construct a limited portion of the full decision tree which characterised the investment problem that they faced.

Under the first maximising strategy (Next Bug), the current bug is offered to the chick whose eating of it would maximise the total weight of all chicks in the nest at the time the next bug is expected to be found. The second strategy (Two Bugs) is identical to the first except that it maximises nest weight at the time the second subsequent bug is expected to be found. The third strategy (1$10 \mathrm{Bugs}$ ) maximizes the short-term expected value of nest weight - that is, the sum, over the next 10 time-steps, of the probability of finding the next food item multiplied by predicted total nest weight at that time-step. This third strategy thus copes with variance in the interval between finding bugs. Surprisingly, not only did all three strategies perform worse than the multi-cue and single-cue rules, but by and large they performed worse than feeding chicks at random. In addition, the most sophisticated optimization strategy out-performed its less complex relatives in only one environment.

These three strategies are far more complex than the successful simple decision rules. They require knowledge that actual parents are unlikely to possess and could not directly assess. They integrate this information in an attempt to determine the best possible decision to make, and yet, despite all that, they make terrible decisions. Why are the simple strategies so much more successful than their complicated, computationally expensive competitors?

A general answer to this kind of problem is that the nature of the provisioning problem faced by many parents involves long-term dependencies which ensure that actions which are successful in the short term may have catastrophic impact in the longer run. In the current context, one form of long-term dependency is the difference between day and night. Even the most far-sighted of the shortterm optimisation rules that we tested could only base its behaviour on what it expected to happen in the next 100 minutes. For most of every day such a strategist plans and acts in blissful ignorance of the coming dusk. Whilst the short-term future anticipated by such a strategist may look bright, the fact that no foraging can be undertaken during the 10 hours of darkness ensures that any optimism may be misguided.

The hypothesis that the poor performance of these short-term optimisers is due entirely to the unforeseen effect of night falling was tested by shortening the simulation's hours of darkness from 10 to zero. As predicted, in this "land of the midnight sun" the performance of the optimising strategies increased, but only to around that of feeding in random order (Fig. 3). In no environment did an optimising strategy outperform the best simple rule. Clearly the parental 

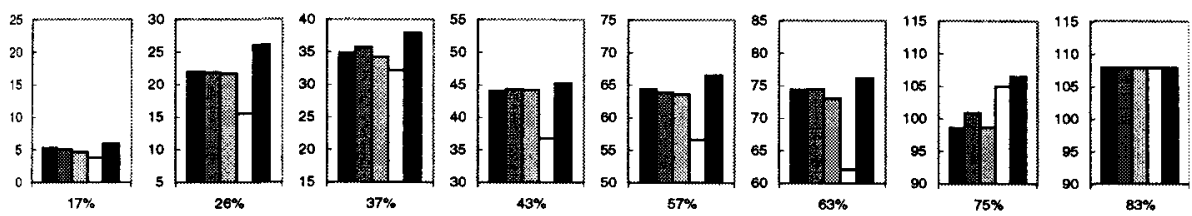

Fig. 3. Columns show the mean nest weight achieved by Random, Next Bug, Two Bugs, 1-10 Bugs, and Best Simple, respectively over 500 runs in eight environments in which each day comprised 14 hours of daylight and an instantaneous period of night.

investment problem features residual long-term dependencies which continue to defeat short-term optimisation.

As these results suggest, the problem is wider than dealing with the cycle of night and day. Many of the decision-making problems faced by humans and other animals exhibit similar kinds of long-term dependencies which invalidate short-term optimisation approaches. The stock market, for example, has proven to be extremely resistant to short-term optimisation approaches largely because of the long-term dependencies and stochasticity which characterise it. However, this does not stop simple rules of thumb such as "only invest in well-known companies" from making money [3].

The failure of short-term optimisation in these contexts might therefore be considered to be symptomatic of a general failure of normative models to apply unproblematically to realistic decision-making problems [20]. Whilst simplified models of these problems may be tractable, adding even the degree of realism modelled here renders them inapplicable in their full-blown form, and hard to approximate using attenuated short-term versions. In this sense, the success of the simple rules which were explored in this study cannot be accounted for by claiming that they approximate some optimal solution to the problem they face, since no such optimal solution, nor any successful approximation to it, can be presented.

\section{Conclusion}

Unlike Becker's unboundedly rational parents, discussed earlier, our results suggest that parents do not have to carry cumbersome investment equations in their heads. The unsophisticated feeding decision rules we present here were successful despite their irrational simplicity. Combined with an understanding of the role of parental egalitarianism, these rules allow parental investment decisions to be robust with respect to changes in environmental quality. Indeed, within our simulation, it is only through the use of a small set of environmentally contingent decision rules that parents can be most successful.

Although empirical studies demonstrate a diversity of parental feeding rules, to date there has been very little information on the relation between these rules and the richness of the environments in which they operate. Our findings suggest 
that the notion of parental egalitarianism coupled with an understanding of the simple rules which parents may use to vary this egalitarianism can be used to better understand this body of empirical data.

The results of this research suggest not only that the state of the environment strongly affects the success of parental investment strategies, but also that even under these complex conditions, strategies do not have to be complex to be successful.

\section{Acknowledgements}

This paper benefited from the comments of Jason Noble, Henrietta Wilson, two anonymous referees, and the programming skills of Martin Dieringer.

\section{References}

1. Kahneman, D., Slovic, P., Tversky, A.: Judgement under uncertainty: Heuristics and biases. CUP, New York (1982)

2. Brooks, R.A.: New approaches to robotics. Science 253 (1991) 1227-1232

3. Gigerenzer, G., Todd, P.M., the ABC Group: Simple heuristics that make us smart. OUP, New York (1999)

4. Webb, B.: A robot cricket. Science 275 (1996) 62-67

5. Green, R.F.: Stopping rules for optimal foragers. Am. Nat. 123 (1984) 30-43

6. Becker, G.S.: A treatise on the family. HUP, Cambridge, MA (1991)

7. Lack, D.: The natural regulation of animal numbers. OUP, Oxford (1954)

8. Parker, G.A.: Models of parent-offspring conflict. V. Effects of the behaviour of the two parents. Anim. Behav. 33 (1985) 519-533

9. Godfray, H.C.J.: Signaling of need by offspring to their parents. Nature 352 (1991) 328-330

10. Winkler, D.W.: A general model for parental care. Am. Nat. 130 (1987) 526-534

11. Horsfall, J.A.: Brood reduction and brood division in coots. Anim. Behav. 32 (1984) 216-225

12. Mondloch, C.J.: Chick hunger and begging affect parental allocation of feeding in pigeons. Anim. Behav. 49 (1995) 601-613

13. Martins, T.L.F., Wright, J.: Brood reduction in response to manipulated brood sizes in the common swift. Behav. Ecol. and Soc. 32 (1993) 61-70

14. Ryden, O., Bengtsson, H.: Differential begging and locomotory behavior by early and late hatched nestlings affecting the distribution of food in asynchronously hatched broods of altricial birds. Zeitschrift für Tierpsychologie 53 (1980) 291303

15. Mock, P.J., Khubesrian, M., Larcheveque, D.M.: Energetics of growth and maturation in sympatric passerines that fledge at different ages. Auk 108 (1991) 34-41

16. Dykstra, C.R., Karasov, W.H.: Daily energy expenditure by nestling house wrens. Condor 95 (1993) 1028-1030

17. Gottlander, K.: Parental feeding behavior and sibling competition in the pied flycatcher, Ficedula hypoleuca. Ornis Scandanavica 18 (1997) 269-276

18. Newton, I.: Feeding and development of the sparrowhawk, Accipiter nisus, nestlings. J. Zool. (London) 184 (1978) 465-487

19. Kölliker, M., Richner, H., Werner, I., Heeb, P.: Begging signals and biparental care: nestling choice between parental feeding locations. Anim. Behav. 53 (1998) 215-222

20. Simon, H.A.: Invariants of human behavior. Ann. Rev. Psych. 41 (1990) 1-19 